\title{
Correction to: Genome size and chromosome number of ten plant species from Kerguelen Islands
}

\author{
Sonja Siljak-Yakovlev ${ }^{1}$ (I) Françoise Lamy ${ }^{1,2} \cdot$ Najat Takvorian $^{1,3} \cdot$ Nicolas Valentin $^{4} \cdot$ Valérie Gouesbet $^{5}$. \\ Françoise Hennion $^{5}$. Thierry Robert ${ }^{1,3}$
}

Published online: 20 November 2020

(c) Springer-Verlag GmbH Germany, part of Springer Nature 2020

\section{Correction to: Polar Biology https://doi.org/10.1007/s00300-020-02755-7}

The original article was published with missing information in Table 1. The complete Table 1 is now provided:

The original article can be found online at https://doi.org/10.1007/ s00300-020-02755-7.

Sonja Siljak-Yakovlev

sonia.yakovlev@u-psud.fr;

sonia.yakovlev@universite-paris-saclay.fr

1 Université Paris-Saclay, CNRS, AgroParisTech, Ecologie Systématique Evolution, 91405 Orsay, France

2 Département de Biologie, 45 bd des Etats-Unis, Université Versailles-Saint-Quentin, 78035 Versailles, France
3 Sorbonne Université, UFR 927, 4 Place Jussieu, 75252 Paris Cedex 05, France

4 Institute of Integrative Biology of the Cell (I2BC), CEA, CNRS, Université Paris-Saclay, 91198 Gif-sur-Yvette, France

5 Ecosystèmes, Biodiversité, Evolution, UMR 6553 ECOBIO, Université de Rennes 1, OSUR, CNRS, 35042 Rennes, France 
Table 1 Origin of investigated populations with localities, GPS coordinates and year of collection

\begin{tabular}{|c|c|c|c|c|}
\hline Species/population & Geographical range & Locality & GPS coordinates & Campaign \\
\hline Colobanthus kerguelensis Hook.f. (Caryophyllaceae) & Endemic to SIOP & & & \\
\hline IB & & Isthme Bas & $-49.3872 ; 70.27326$ & 2016-2017 \\
\hline CAJ & & Cabane Jacky & $-49.3134 ; 70.1234$ & 2016-2017 \\
\hline TUS & & Plateau du Tussok & $-49.2967 ; 70.04608$ & 2016-2017 \\
\hline RBA-10 & & Péninsule Rallier du Baty & $-49.6601 ; 68.9797$ & $2017-2018$ \\
\hline $\mathrm{SUZ}^{*}$ & & Pointe Suzanne & n.a. & April 2017 \\
\hline $\begin{array}{l}\text { Limosella australis R.Br. } \\
\text { (Scrophulariaceae) }\end{array}$ & Wide, native & & & \\
\hline PJDA3 & & Presqu'île Jeanne d'Arc & $-49.52387 ; 70.15679$ & 2015-2016 \\
\hline Lyallia kerguelensis Hook.f. (Montiaceae) & Strict endemic & & & \\
\hline AUS-23 & & South of Ile Australia & $-49.4646 ; 69.8769$ & 2015-2016 \\
\hline MAY-2 & & Ile Mayes & $-49.47404 ; 69.93306$ & 2016-2017 \\
\hline MAC-3 & & Ile Mac Murdo & $-48.89503 ; 69.41626$ & $2017-2018$ \\
\hline AUS- $25^{*}$ & & South of Ile Australia & $-49.46568 ; 69.87563$ & March 2019 \\
\hline AUS- $30^{*}$ & & South of Ile Australia & $-49.47672 ; 69.89263$ & March 2019 \\
\hline Poa aпnиa L. (Poaceae) & Wide, introduced & & & \\
\hline AUS-N & & North of Ile Australia & $-49.43579 ; 69.84936$ & 2016-2017 \\
\hline $\mathrm{PAF}^{*}$ & & Port-aux-Français & $-49.3526 ; 70.2170$ & 2016-2017 \\
\hline STU-7 & & Val Studer & n.a. & 2015-2016 \\
\hline MOL & & Pointe Molloy & $-49.3523 ; 70.07608$ & 2016-2017 \\
\hline P. cookii (Hook.f.) Hook.f. & Endemic to SIOP & & & \\
\hline AUS-N & & North of Ile Australia & $-49.4387 ; 69.8496$ & 2016-2017 \\
\hline MAC 4 & & Ile Mac Murdo & $-48.8877 ; 69.4199$ & $2017-2018$ \\
\hline AUS-S & & South of Ile Australia & $-49.47223 ; 69.89118$ & 2015-2016 \\
\hline P. kerguelensis (Hook.f.) Steud. & Endemic to SIOP & & & \\
\hline AUS-N & & North of Ile Australia & $-49.43973 ; 69.85439$ & 2016-2017 \\
\hline IB & & Isthme Bas & n.a. & 2016-2017 \\
\hline PAF & & Near Port-aux-Français & $-49.3227 ; 70.1768$ & 2016-2017 \\
\hline $\mathrm{CAJ}$ & & Cabane Jacky & $-49.3132 ; 70.1233$ & 2016-2017 \\
\hline Pringlea antiscorbutica R.Br. ex Hook.f. (Brassicaceae) & Endemic to SIOP & & & \\
\hline AUS-N & & North of Ile Australia & $-49.43763 ; 69.84476$ & 2016-2017 \\
\hline $\mathrm{CAJ}$ & & Cabane Jacky & $-49.3124 ; 70.1239$ & 2016-2017 \\
\hline TUS & & Plateau du Tussok & n.a. & 2016-2017 \\
\hline $\begin{array}{l}\text { Ranunculus biternatus } \mathrm{Sm} . \\
\text { (Ranunculaceae) }\end{array}$ & Circumpolar area & & & \\
\hline AUS-N & & North of Ile Australia & $-49.43211 ; 69.83792$ & 2016-2017 \\
\hline IB-13 & & Isthme Bas & $-49.3818 ; 70.2752$ & 2016-2017 \\
\hline IB-2 & & Isthme Bas & $-49.3843 ; 70.2590$ & 2016-2017 \\
\hline R. moseleyi Hook.f. & Strict endemic & & & \\
\hline AUS-N5 & & North of Ile Australia & $-49.4216 ; 69.8239$ & 2016-2017 \\
\hline IB-2 & & Isthme Bas & $-49.3843 ; 70.2590$ & 2016-2017 \\
\hline PAF-5 & & Port-aux-Français & $-49.3352 ; 70.2265$ & 2016-2017 \\
\hline R. pseudotrullifolius Skottsb. & Magellanic and Kerguelen & & & \\
\hline AUS-N6 & & North of Ile Australia & $-49.4497 ; 69.8642$ & 2016-2017 \\
\hline IB-1 & & Isthme Bas & $-49.3849 ; 70.2752$ & 2016-2017 \\
\hline OTA-1 & & Mouth of Rivière des Otaries & $-49.3456 ; 70.1654$ & 2016-2017 \\
\hline
\end{tabular}

Site abbreviations: AUS Ile Australia, CAJ Cabane Jacky, IB Isthme Bas, MAY Ile Mayes, MAC Ile Mac Murdo, MOL Pointe Molloy, OTA mouth of Rivière des Otaries, PAF Port-aux-Français, PJDA Presqu'île Jeanne d'Arc, RBA Péninsule Rallier du Baty. STU Val Studer. SUZ Pointe Suzanne, TUS Plateau du Tussok. Name of site is followed by population number. SIOP South Indian Ocean Province

*Population used only for chromosome counting

This correction stands to correct the original article.

Publisher's Note Springer Nature remains neutral with regard to jurisdictional claims in published maps and institutional affiliations. 